\title{
Efficient Resource Allocation of Service Differentiated Multiuser MIMO Systems Using Power Control
}

\author{
S. Balaji ${ }^{1}$, L. Nithyanandan ${ }^{2}$ and P. S. Mallick ${ }^{1}$ \\ ${ }^{1}$ School of Electrical Engineering, VIT University, \\ Vellore-632014, India \\ ${ }^{2}$ Pondicherry Engineering College, Puducherry-605014, India \\ E-mail: \{sbalaji; psmallick\}@vit.ac.in; nithi@pec.edu
}

Received 2 April 2016; Accepted 15 May 2016;

Publication 4 August 2016

\begin{abstract}
This paper considers power allocation across MIMO channels for transmitting two different Classes of Service. MIMO provides multiple parallel channels and it transmits different types of services separately because of different Quality of Service requirements. A high priority class of service named as Constant Bit Rate Traffic has limited delay constraints. So priority is given to this traffic in power allocation and then remaining power is allocated to other services i.e. here Best Effort Traffic based on water-filling algorithm. Analytical expressions are presented to assess the outage probability in terms of average access time and the effect of channel estimation error in throughput is also studied. Numerical results and Analysis are also provided to evaluate the Performance indices like outage probability, throughput and probability of access of both classes of service by optimally allocating the power. The Analysis is carried out for uncorrelated Rayleigh-fading channels with and without interference.
\end{abstract}

Keywords: Power allocation, Outage Probability, Throughput, Probability of Access, MIMO.

Journal of Green Engineering, Vol. 6, 27-50.

doi: 10.13052/jge1904-4720.612

(c) 2016 River Publishers. All rights reserved. 


\section{Introduction}

The demand for data bandwidth has increased enormously; the next generation wireless networks adopted widely the Multiple Input Multiple Output (MIMO) standards to cater the bandwidth requirement. The capacity analysis of MIMO channels under rich scattering environment has been investigated $[1,2]$ to explore the achievable rates of the channels. In [3] delay limited capacity of MIMO system is derived and in [4] the analysis of capacity with channel fading correlations is carried out. There are different approaches to characterize the performance of MIMO channels based on the availability of instantaneous or statistical channel state information (CSI). When the CSI is perfectly available at the both transmitter and receiver it is called as perfect CSI and if the CSI is known only at receiver it is called as partial CSI. The capacity scaling laws are derived for partial CSI case in MIMO environment [5]. The capacity constraints on both cases of CSI have been discussed extensively in the past by many research works.

The effect of spatial multiplexing of MIMO is considered in [6-9] and especially the effect of power allocation on resource allocation is considered in $[6,7]$. Usually MIMO Provides two types of gain to achieve fair power allocation i.e. one is the spatial multiplexing (SM) gain and other is the diversity gain. The trade-off between these two types of gain has been studied in detail in [8]. In general, the resource allocation of MIMO systems widely varying with number of transmit and or receive antennas, target signal to noise ratio (SNR), transmission schemes, correlation among channel coefficients, etc. Thus the accurate quantification of these parameters is required to achieve the optimal performance.

Power allocation for SM in MIMO channels is an important issue in order to improve the performance of MIMO. The power allocation using beamforming between inter-cluster and intra-cluster Networks is also analysed in detail [10] and closed form expressions are derived for Quality of Service (QoS) driven power allocation to maximize the throughput for wireless networks [11]. Power efficiency maximization in multicell cooperation networks for provisioning diverse QoS is also explored [12]. The power allocation for MIMO systems by taking into account the channel estimation error is also reported in [13]. When the minimum power requirement is specified, the closed form expressions for Average Level Crossing rate (LCR), average outage duration (AOD) are derived for various fading environments [14]. The Time varying performance analysis of wireless networks with constant rate traffics has also been proposed in the past and power allocation in cooperative communication for Best effort delivery has also been discussed $[15,16]$. 
The power allocation with imperfect CSI for interference channels has also been analysed [17]. The issue of stability and queuing delay for bursty traffic is addressed in detail for simultaneous transmission with multiple antennas [18]. While dealing the queuing and scheduling of users, the important properties of average waiting time and access time with emphasis on channel dynamics is studied [19]. These properties are very important in scheduler design especially if the design is delay sensitive. The scheduling in MIMO systems, with large set of users with different classes of service requirements is also analysed [20] and analytical framework for scheduling in MIMO with imperfect CSI has also been addressed [21, 22]. Our intuition is to propose a robust power allocation for different priority based traffics in MIMO systems and to compare the performance metrics in both interference and non-interference regimes. Our contributions are summarized below.

In this work, we propose the power allocation for MIMO block-fading channels in the presence of two different types of traffics, i.e., Constant Bit Rate (CBR) and best-effort (BE) traffics. We derived the expression for outage probability of the CBR users by considering the effect of queuing delay and average access time of the user. Expressions for probability of access of the user are presented if the user is affected by interference from remaining users. We also evaluated the effect of channel estimation error on the throughput of Best Effort users and also throughput improvements are investigated in interference and non-interference regimes.

The rest of the paper is organized as follows. In Section 2, the system model and proposed power allocation methodology is described and in Section 3, the outage probability of Constant Bit Rate Traffic is described in both interference and non-interference regimes and Section 4 describes the throughput analysis of best effort traffic in both scenarios. Section 5 theoretical results are corroborated with analytical evaluations and in Section 6 concluding remarks are given.

\section{System Model}

In MIMO systems, according to the data rate that is decided by optimal power allocation, single data or multiple data are multiplexed and transmitted through the parallel channels. If there are multiple data streams and if each have different delay requirement, then those data streams have to be dealt differentially. In constant bit rate service a constant data rate should be maintained in order to meet the stringent delay requirement. There is no condition of stringent delay requirement as far as other data traffics are 
concerned. For example, in Best effort traffic, throughput has to be maintained without maintaining the delay requirement. To satisfy the requirement of both $\mathrm{CBR}$ and $\mathrm{BE}$, the system needs to designed properly. The block diagram of MIMO transmission to support different data streams is shown in the Figure 1.

The system considered to be consisting of $N_{r}$ receiving antennas and $N_{t}$ transmitting antennas. The Number of transmitting antennas is greater than the number of receiving antennas and the elements of the channel matrix $H$ are identically independently distributed. The channel matrix $H$ is assumed to be invariant for a frame duration i.e. the channel is of block fading type. Let the receiver is interested in decoding the $l^{t h}$ transmitted vectors as [7],

$$
y_{l}=H s_{l}+n_{l}, l=0,1, \ldots . M-1,
$$

Where $y_{l}, s_{l}$ and $n_{l}$ denote the received signal vector, the transmitted signal vector, and the background noise vector respectively. The length of the frame is denoted by $M$. The noise vectors $\eta_{l}$ assumed to be Gaussian and are an independent circular complex random vector with the following properties

$$
E\left[n_{l}\right]=0, E\left[n_{l} n_{l}^{H}\right]=N_{0} I, E\left[n_{l} n_{l}^{T}\right]=0
$$

where $N_{0}$ denotes the double-sided noise spectral density. The total transmission power $E\left[s_{l}^{2}\right]$ is constrained as [7]

$$
E\left[s_{l}^{2}\right] \leq P_{\text {Total }}
$$

where $P_{\text {Total }}$ denotes the total power and the input signal to noise ratio (SNR) is denoted by $S N R_{\text {in }}=\frac{P_{\text {Total }}}{N_{0}}$.

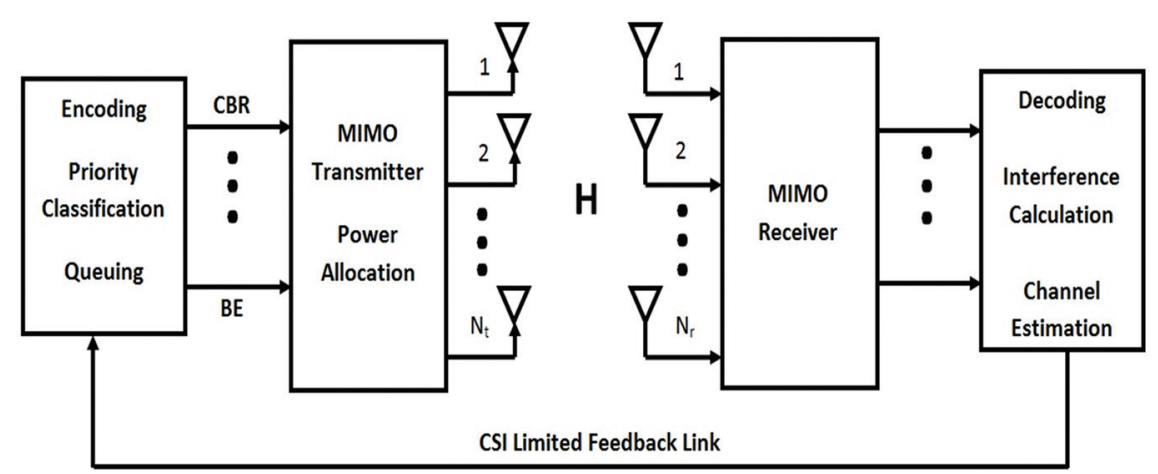

Figure 1 Block diagram for MIMO systems. 
Since multiple traffics have to be transmitted simultaneously, the MIMO channel between transmitter and receiver should be decomposed to provide multiple parallel paths. Varieties of methods are available to decompose the channel matrix $H$ of Equation (1) and most widely, versatile used methods are singular value decomposition (SVD) [23] and QR factorization [25]. Using QR factorization, the channel $H$ is decomposed as $H=Q R$ where $\mathrm{Q}$ is unitary matrix and $\mathrm{R}$ is the upper triangular matrix. By applying the $\mathrm{QR}$ decomposition, the received vector of Equation (1) with $Q^{H}$ is

$$
Z_{l}=R s_{l}+\overline{n_{l}}
$$

where $R=Q^{H} H$ and $\overline{n_{l}}=Q^{H} n_{l}$. The class (Traffic) of service called Constant bit rate (CBR) Traffic is assumed in this paper as A. This class of service given to the users whose traffics are delay sensitive. Because of its delay constraint, the users of CBR traffic are given priority over other class users in power allocation, channel feedback and scheduling. The other class of service considered in this paper is Best Effort (BE) traffic and it is denoted as B. There is no definite quality of service requirement for these users. Therefore their traffic is based on Signal to noise ratio and the interference they cause to other users. There are $K$ users in the system, $K_{A}$ and $K_{B}$ refers to the users belonging to the category of CBR and BE Traffics. The proposed power allocation with power constraints is described next.

Since there are $N_{t}$ antennas and after applying QR decomposition, there are assumed to be $N_{t}$ sub channels are available. The SNR's of each channel are represented as $\gamma_{1}, \gamma_{2} \ldots \ldots, \gamma_{N_{t}}$ and each SNR's are feedback to the transmitter via backhaul link. In general, the SNR's are expressed [7] as

$$
\gamma_{n_{t}}=\frac{\left|r_{n_{t}, n_{t}}\right|^{2}}{N_{o}}
$$

where $r_{n_{t}, n_{t}}$ denotes the $n_{t}^{\text {th }}$ diagonal element of $\mathrm{R}$ in QR decomposition of Equation (4). The power allocation problem can be decomposed into the following two sub problems. Since Constant Bit Rate traffics which have higher priority than best-effort traffics, we need to allocate power first to CBR and then to BE.

In our proposed model in order to reduce the queuing delay and to avoid packet loss, some minimum amount of power is allocated to serve BE users. This is done by defining power allocation ratio $\alpha$ which is the ratio of allocated BE power to CBR power i.e. $\alpha=\frac{P_{B E}}{P_{C B R}}$. In this proposed power allocation methodology the value of $\alpha$ lies between 0 and 1 i.e. suppose sufficient CBR 


\section{S. Balaji et al.}

users are having no data to send then $\alpha$ can take the maximum value as one $\left(P_{B E}=P_{C B R}\right)$. This allocation of maximum $P_{B E}$ is equal to $P_{C B R}$ is considered to satisfy the demands of high priority delay sensitive applications in a better way compared to low priority applications and moreover in a real time practical environment delay prone applications out numbering the delay insensitive ones. In rare situations, if $P_{C B R}$ is $0, \alpha$ tends to infinity. If $\alpha$ goes to infinity, entire total power will be allocated to $P_{B E}$ and in reality this will rarely happen. Thus the proposed methodology ensures minimum allocation to $\mathrm{BE}$ while maintaining guaranteed QoS.

For Constant Bit Rate traffics, the set of sub channels A, that minimizes the power can be found as

$$
\begin{aligned}
& \min \sum_{n_{t} \in N t} P_{\text {Total }} \\
& \text { subject to } P_{C B R}+P_{B E}=P_{\text {Total }} \\
& P_{C B R}=\frac{P_{\text {Total }}}{1+\alpha}, \quad P_{B E}=\alpha P_{C B R}
\end{aligned}
$$

The power allocated to the sub-channels of CBR traffic should be less than or equal to the target SNR in order to meet the minimum outage constraint. Thus

$$
P_{n_{t}} \gamma_{n_{t}}=\gamma_{C B R}, n_{t} \in A
$$

where $\gamma_{C B R}$ is the target SNR for each sub channel transmitting Constant Bit Rate traffic at a certain constant rate which might be smaller than the fixed channel capacity. Let $P_{A}=P_{C B R}=\sum_{n_{t} \in A} P_{n_{t}}$ denote the total allocated power

to the sub channels for Constant Bit Rate traffics. If $P_{C B R}>\frac{P_{T o t a l}}{1+\alpha}$, some packets of Constant Bit Rate traffics cannot be delivered without errors. Thus the probability of $P_{C B R}>\frac{P_{T \text { otal }}}{1+\alpha}$ is the outage probability for Constant Bit Rate traffics. Once the power $P_{C B R}$ is allocated to Constant Bit Rate traffics, the rest of the power, $P_{B E}=\max \left(P_{\text {Total }}-P_{C B R}, 0\right)=\max \left(P_{\text {Total }}-P_{A}, 0\right)$ is allocated to best-effort traffics with set of sub channels $B=\left\{1,2, \ldots N_{t}\right\} / A$. Then given a power $P_{B E}$, we need to distribute the power across all the sub channels in B optimally. The power allocation problem to best-effort traffics is given as follows.

By the same procedure as explained for CBR traffic, the power allocated to the sub-channels of BE traffic should be less than or equal to the target SNR of each BE channels in order to meet the Maximum throughput. Thus

$$
P_{n_{t}} \gamma_{n_{t}}=\gamma_{B E}, n_{t} \in B
$$


where $\gamma_{B E}$ is the target SNR for each sub channel transmitting Best Effort traffic at a certain constant rate which might be smaller than the fixed channel capacity. Let $P_{B}=P_{B E}=\sum_{n_{t} \in B} P_{n_{t}}$ denote the total allocated power to the sub channels for Constant Bit Rate traffics. If $L$ denotes the number of the sub channels for Constant Bit Rate traffics, then $N_{t}-L$ sub channels are available for Best Effort Traffic and in our proposed model the power allocated to BE users is $P_{B E}=\alpha P_{C B R}$ and by making $P_{B E}$ will never greater than $P_{C B R}$ by suitably adjusting the power allocation ratio $\alpha$. If allocated power is not sufficient for both $P_{C B R}$ and $P_{B E}$, some users of both CBR and BE should be in queue. However in practice the queue length is finite and there would be loss. To decide the queue length with low loss, throughput of best-effort traffics has to be known. The necessary closed form expressions are derived in the following sections.

\section{Outage Probability of CBR}

In this section, the performance analysis of the power allocation method for CBR is derived. The SNR of the each user is a chi-square random variable and the importance performance metric for the Constant Bit Rate traffic is the outage probability and hence $P_{C B R}=\frac{P_{T o t a l}}{1+\alpha}$, and the remaining power i.e. $P_{C B R}=P_{\text {Total }}-P_{B E}$ which produces power allocation for best effort traffics. As already said earlier, the Constant Bit Rate traffics have higher priority than the best-effort traffics, the index set A for Constant Bit Rate traffics can be determined first. Let $\gamma_{u(1)} \geq \gamma_{u(2)} \geq \ldots \ldots \geq \gamma_{u\left(N_{t}\right)}$ be their ordered set of SNR of each layer after QR decomposition. From the ordered set of SNR of received $N_{t}$ channels, the outage probability and throughput are calculated. For an exact performance analysis, order statistics of $\gamma_{n}$ are required. Using ordered $\gamma_{n}$ of the channels, first the outage probability in the absence of interference is derived.

\subsection{In the Absence of Interference}

To study the performance analysis of CBR users, we assume that the signals are detected without interference by employing successive interference cancellation (SIC). In SIC, the signals of lowest layer is detected and decoded and then subsequently successive layers are detected and cancelled. The probability that the power allocated to the CBR users when there are $L$ channels available to the CBR users is 


$$
\begin{aligned}
\operatorname{Pr} & \left.P_{C B R} \leq y\right)=\operatorname{Pr}\left(\frac{P_{\text {Total }}}{1+\alpha} \leq y\right) \\
& =\operatorname{Pr}\left(\frac{\left(P_{1}+P_{2}+P_{3}+\ldots+P_{L}\right)}{1+\alpha} \leq y\right) \\
& =\operatorname{Pr}\left(\frac{\gamma_{C B R}\left(\frac{1}{\gamma_{1}}+\frac{1}{\gamma_{2}}+\frac{1}{\gamma_{3}}+\ldots+\frac{1}{\gamma_{L}}\right)}{1+\alpha} \leq y\right) \\
& =\operatorname{Pr}\left(\frac{1}{L(1+\alpha)}\left(\frac{1}{\gamma_{1}}+\frac{1}{\gamma_{2}}+\frac{1}{\gamma_{3}}+\ldots+\frac{1}{\gamma_{L}}\right) \leq \frac{y}{L \gamma_{C B R}}\right) \\
& =\operatorname{Pr}\left(H \geq \frac{L(1+\alpha) \gamma_{C B R}}{y}\right)
\end{aligned}
$$

where $H^{-1}=\frac{1}{L}\left(\frac{1}{\gamma_{1}}+\frac{1}{\gamma_{2}}+\frac{1}{\gamma_{3}}+\ldots+\frac{1}{\gamma_{L}}\right)$. By incorporating arithmetic and harmonic mean inequality,

$$
\operatorname{Pr}\left(P_{C B R} \leq y\right)=\operatorname{Pr}\left(\gamma_{1}+\gamma_{2}+\ldots+\gamma_{L} \geq \frac{L^{2}(1+\alpha) \gamma_{C B R}}{y}\right)
$$

where the sum $\gamma_{1}+\gamma_{2}+\ldots+\gamma_{L}$ is a chi-square random variable. From [7], an approximation of $\operatorname{Pr}\left(P_{C B R} \leq y\right)$ of Equation (14) can be written as

$$
\operatorname{Pr}\left(\mathrm{P}_{C B R} \leq \mathrm{y}\right)=1-\frac{\gamma\left(\mathrm{v}, \frac{\mathrm{L}^{2}(1+\alpha) \gamma_{C B R}}{\mathrm{y}}\right)}{\Gamma(\mathrm{v})}
$$

where $\gamma(k, z)$ is the incomplete gamma function i.e. $\gamma(k, z)=\int_{0}^{z} t^{k-1} e^{-t} d t$ and $\Gamma(v)$ is the gamma function and $v=\sum_{n=1}^{L}\left(N_{r}-n+1\right)$. From Equation (15), the approximated outage probability is

$$
\operatorname{Pr}_{\text {out }}=1-\operatorname{Pr}\left(P_{C B R} \leq P_{\text {Total }}\right)=\frac{\gamma\left(v, \frac{L^{2}(1+\alpha) \gamma_{C B R}}{P_{\text {Total }}}\right)}{\Gamma(v)}
$$

As stated already, after the assumption of sufficiently and statically random channel matrix, the channel capacity of CBR becomes [24]

$$
C=N_{\min } \frac{1}{2} \log _{2}\left(1+\frac{2 \gamma_{C B R} N_{r}}{N_{\min }}\right)
$$


The signal to noise ratio from the above equation is obtained as

$$
\gamma_{C B R}=\frac{N_{\min }}{2 N_{r}}\left(2^{\frac{2 c}{N_{\min }}}-1\right)
$$

where $N_{\min }$ is the minimum of number of transmitting and receiving antennas in the system. The Equation (18) is substituted in Equation (16) and the probability of outage is obtained as

$$
P r_{\text {out }}=\frac{\left(v, \frac{L^{2}(1+\alpha) \frac{N_{\text {min }}}{2 N_{r}}\left(2^{\frac{2 c}{N_{\min }}}-1\right)}{P_{\text {Total }}}\right)}{\Gamma(v)}
$$

From (19) we can see that outage probability decreases if (i) the total transmitted power increases; (ii) the number of channels $L$ increases and; (iii) target SNR increases. This is merely because of increase of $L$ and target SNR requires more power for a successful transmission when total power is fixed.

\subsection{In Interference}

To satisfy the power requirement of CBR users in the presence of interference, outage probability is derived with probability of user access. Each $K_{A}$ user is assumed to encounter interference from remaining $K_{A}-1$ users. The interference average power $\Omega_{i}$, fade of the desired user $\Omega_{D}$, the Doppler frequency $f_{m}$, probability that the signal falls below a predefined threshold $P_{o}$ and average level crossing rate $\eta$ is related by [20]

$$
\begin{aligned}
P_{o} & =1-\frac{1}{\left(1+\frac{\gamma_{C B R} \Omega_{i}}{\Omega_{\mathrm{D}}}\right)^{K_{A}-1}} \\
\eta & =\frac{\sqrt{2 \pi} f_{m} \Gamma\left(K_{A}-\frac{1}{2}\right)}{\Gamma\left(K_{A}-1\right)}\left(\frac{1}{1+\frac{\gamma_{C B R} \Omega_{i}}{\Omega_{\mathrm{D}}}}\right)^{K_{A}-1} \sqrt{\frac{\gamma_{C B R} \Omega_{i}}{\Omega_{\mathrm{D}}}}
\end{aligned}
$$

In order to reduce the interference, the probability that the user access the channel $p_{i}$ should be less. The average access time of the user is given by 


\section{S. Balaji et al.}

$T_{i}=\frac{p_{i}}{R_{i}}$ where $i=0,1,2, \ldots, L$ and $R_{i}$ is the average access rate of the user. Since this average access time should be greater than or equal to the time the scheduler can wait till it schedule the user. Therefore the average access time of the user should be greater than the sum of outage time $T_{o}$ and queuing time $T_{Q}$ i.e.

$$
T_{i} \geq T_{Q}+T_{o}
$$

Substituting $T_{Q}$ and $T_{\text {out }}$ in the above equations

$$
\begin{aligned}
& \frac{p_{i}}{R_{i}} \geq \frac{1}{\mu_{A} K_{A}-\lambda_{A}}+\frac{P_{o}}{\eta} \\
& p_{i} \geq R_{i}\left(\frac{1}{\mu_{A} K_{A}-\lambda_{A}}+\frac{P_{o}}{\eta}\right)
\end{aligned}
$$

where $\mu_{A}$ and $\lambda_{A}$ are average packet arrival and average service time of CBR traffic. The closed form expression for $R_{i}$ is given by (for Rayleigh fading case) [19]

$$
R_{i}=\pi f_{m}(L-1) \sum_{n=0}^{L-2}\left(\begin{array}{c}
L-2 \\
n
\end{array}\right)(-1)^{n}(n+2)^{\frac{-3}{2}}
$$

The value of $\frac{P_{o}}{\eta} \times R_{i}$ is obtained as

$$
\begin{aligned}
\frac{P_{o}}{\eta} \times R_{i}= & \frac{1-\frac{1}{\left(1+\frac{\gamma_{C B R} \Omega_{i}}{\Omega_{\mathrm{D}}}\right)^{K_{A}-1}}}{\frac{\sqrt{2 \pi} f_{m} \Gamma\left(K_{A}-\frac{1}{2}\right)}{\Gamma\left(K_{A}-1\right)}\left(\frac{1}{1+\frac{\gamma_{C B R} \Omega_{i}}{\Omega_{\mathrm{D}}}}\right)^{K_{A}-1} \sqrt{\frac{\gamma_{C B R} \Omega_{i}}{\Omega_{\mathrm{D}}}}} \\
& \times \pi f_{m}(L-1) \sum_{n=0}^{L-2}\left(\begin{array}{c}
L-2 \\
n
\end{array}\right)(-1)^{n}(n+2)^{\frac{-3}{2}}
\end{aligned}
$$

By applying Kershaw's law of inequality for the gamma function i.e. $\frac{\Gamma(x+\mathrm{s})}{\Gamma(x+1)}<\left(\mathrm{x}+\frac{\mathrm{s}}{2}\right)^{s-1}$, for all $x>0,0<s<1, \frac{\Gamma\left(K_{A}-\frac{1}{2}\right)}{\Gamma\left(K_{A}-1\right)}$ in the denominator of the above equation is simplified as

$$
\frac{\Gamma\left(K_{A}-\frac{1}{2}\right)}{\Gamma\left(K_{A}-1\right)}<\left(K_{A}-\frac{5}{4}\right)^{\frac{1}{2}}
$$


After applying the above inequality and using the basic property of $(1+x)^{2 n} \geq$ $1+2 n x$ for $x \in R, n \in N$, we can write the Equation (26) as

$$
\begin{aligned}
\frac{P_{o}}{\eta} \times R_{i}= & \frac{\left(\left(K_{A}-1\right) \sqrt{\frac{\gamma_{C B R} \Omega_{i}}{\Omega_{\mathrm{D}}}}\right)}{\sqrt{2 \pi}\left(K_{A}-\frac{5}{4}\right)^{\frac{1}{2}}} \\
& \times \pi(L-1) \sum_{n=0}^{L-2}\left(\begin{array}{c}
L-2 \\
n
\end{array}\right)(-1)^{n}(n+2)^{\frac{-3}{2}}
\end{aligned}
$$

Substituting the above Equation (28) in Equation (24), the probability of user access is reduced to

$$
\begin{aligned}
p_{i} \geq & \left(\frac{f_{m}}{\mu_{A} K_{A}-\lambda_{A}}+\frac{\left(\left(K_{A}-1\right) \sqrt{\frac{\gamma_{C B R} \Omega_{i}}{\Omega_{\mathrm{D}}}}\right)}{\sqrt{2 \pi}\left(K_{A}-\frac{5}{4}\right)^{\frac{1}{2}}}\right) \\
& \times \pi(L-1) \sum_{n=0}^{L-2}\left(\begin{array}{c}
L-2 \\
n
\end{array}\right)(-1)^{n}(n+2)^{\frac{-3}{2}}
\end{aligned}
$$

In the interference system, if the normalized SNR is greater than or equal to the target SNR, the access probability is related with the number of channels as

$$
p_{i}=\operatorname{pr}\left(P_{i} \gamma_{i} \geq \gamma_{C B R}\right)=\frac{1}{L}
$$

Substituting the above Equation (30) in Equation (19) and calculating using minimum probability of access, the probability of outage is expressed as

$$
P r_{\text {out }}=\frac{\gamma\left(v, \frac{\frac{N_{\text {min }}}{2 N_{r}}(1+\alpha)\left(2^{\frac{2 c}{N_{\text {min }}}}-1\right)}{\left(p_{i}\right)^{2} P_{\text {Total }}}\right)}{\Gamma(v)}
$$

From the above Equation (31), the outage probability is a function of power allocation ratio $\alpha$ and probability of user access. If the probability of user access increases, it is expected that the outage probability decreases. The change in outage probability is demonstrated in results and discussion section. 


\section{Throughput Analysis for Best-Effort Traffics}

Throughput which is the average capacity of the remaining $N_{t}-L$ sub channels with optimum power allocation. First the throughput of the BE traffic in the absence of interference is derived. If $L=0$, the throughput is identical to the Ergodic capacity and optimum power allocation is known at transmitter. The conditional throughput for best-effort traffics is given by [7]

$$
T_{B E}=E\left[\max _{\sum_{l=L+1}^{N_{t}} P_{B E}} \sum_{l=L+1}^{N_{t}} \log _{2}\left(1+P_{l} \gamma_{l}\right)\right]
$$

The throughput is expectation of $T_{B E}$ over Power allocated to best-effort traffics and is given as

$$
\begin{aligned}
T_{B E} & \leq E\left[\max _{\substack{N_{t} P_{l} \leq P_{B E} \\
l=L+1}} \sum_{l=L+1}^{N_{t} \geq 0} \log _{2}\left(1+P_{l} \gamma_{l}\right)\right] \\
& =E\left[\sum_{l=L+1}^{N_{t}} \log _{2}\left(1+P_{l} \gamma_{l}\right)\right]
\end{aligned}
$$

By using the fact that the optimum power according to water filling theorem is given in [23] as $P_{l}=\left(\beta-\frac{1}{\gamma_{l}}\right)^{+}, l=L+1, L+2, \ldots \ldots N_{t}$ and the expression inside the log function becomes

$$
\log _{2}\left(1+P_{l} \gamma_{l}\right)=\log _{2}\left(1+\beta \gamma_{l}\right)
$$

where $\beta$ is the Lagrangian multiplier. By utilizing the Lagrangian multiplier, the throughput becomes

$$
T_{B E}=\sum_{l=L+1}^{N_{t}} E\left[\log _{2} \beta+\log _{2} \gamma_{l}\right]
$$

In the proposed power allocation of best effort traffic, the total power allocated to the Best Effort traffic is the sum of all $P_{l}$ and the sum is

$$
P_{B E}=\sum_{l=L+1}^{N_{t}} P_{l}=\sum_{l=L+1}^{N_{t}}\left(\beta-\frac{1}{\gamma_{l}}\right)^{+}
$$


If $P_{l}>0$, for $l=L+1, L+2, \ldots \ldots K, \beta$ becomes

$$
\beta \triangleq \frac{1}{N_{t}-L}\left(P_{B E}+\sum_{l=L+1}^{N_{t}} \frac{1}{\gamma_{l}}\right)
$$

From the Equation (38), the Lagrangian parameter for the power allocation to the best effort traffic can be calculated.

The Channel condition(CSI) of the Best Effort user is utmost important, since the Best Effort Traffic users have less priority compared to the CBR users and also the channels allocated to the BE users is the remaining channels after allocating to the CBR users. If there is any degradation in CSI at the transmitter, some of the BE users may not be scheduled at all. In our proposed method, the power allocation and scheduling of users when operated in best effort traffic depend on the channel condition of the MIMO at either the transmitter or receiver. Thus Channel information knowledge of the MIMO system will affect the power allocation strategy.

Assume that the channel is estimated and the feedback is given through the feedback link and the channel estimation error results in an SNR loss factor. The SNR loss factor which is related with channel estimation mean square error $\sigma_{E}^{2}$ is obtained as [13]

$$
\delta=\frac{\left(1-\sigma_{E}^{2}\right)}{\left(\left(1+\sigma_{E}^{2} P_{\text {Total }}\right)\right)}
$$

From above equation, the total power in terms of channel estimation error is

$$
P_{\text {Total }}=\left(\frac{\left(1-\sigma_{E}^{2}\right)}{\delta}-1\right) *\left(\frac{1}{\sigma_{E}^{2}}\right)
$$

Substituting the above Equation (40) in the Equation (6) and solving for $P_{B E}$, the power allocated to BE traffic is reduced to

$$
P_{B E}=\left(\left[\frac{\alpha}{1+\alpha}\left(\frac{\left(1-\sigma_{E}^{2}\right)}{\delta}-1\right) *\left(\frac{1}{\sigma_{E}^{2}}\right)\right]\right)
$$

From the above equation, the power allocated to the Best effort traffic is calculated and the value of $P_{B E}$ should be less than or equal to $P_{T o t a l}-P_{C B R}$. The value of $P_{B E}$ is substituted in the Equation (38) and subsequently the Lagrangian multiplier is calculated for the best effort traffic as 


$$
\beta=\frac{1}{N_{t}-L}\left(\left(\left[\frac{\alpha}{1+\alpha}\left(\frac{\left(1-\sigma_{E}^{2}\right)}{\delta}-1\right) *\left(\frac{1}{\sigma_{E}^{2}}\right)\right]\right)+\sum_{l=L+1}^{N_{t}} \frac{1}{\gamma_{l}}\right)
$$

Substituting the above Equation (42) in Equation (36) and using the upper bound from [16], the expected value i.e. $E\left[\log _{2} \gamma_{l}\right]=\left(\log _{2} e\right) \varphi\left(N_{r}-l+1\right)$, the throughput reduces to

$$
\begin{aligned}
T_{B E}= & \log _{2}\left[\frac { 1 } { N _ { t } - L } \left(\left(\left(\left[\frac{\alpha}{1+\alpha}\left(\frac{\left(1-\sigma_{E}^{2}\right)}{\delta}-1\right) *\left(\frac{1}{\sigma_{E}^{2}}\right)\right]\right)\right)\right.\right. \\
& \left.\left.+\sum_{l=L+1}^{N_{t}} \frac{1}{\gamma_{l}}\right)\right]+\sum_{l=L+1}^{N_{t}}\left(\log _{2} e\right) \varphi\left(N_{r}-l+1\right)
\end{aligned}
$$

where $\varphi\left(N_{r}-l+1\right)$ is digamma function. The throughput is a function of $N_{t}, N_{r}$, the number of channels $L$, and the SNR loss factor $\delta$, the power allocation ratio $\alpha$. The variations of throughput with respect different antenna combinations are demonstrated in the results and discussion Section 5.

\subsection{In Interference}

In this section the throughput of the Best effort traffic is derived when the system is affected by the strong interference. From Equation (29), the probability of access of best effort users is written as

$$
\begin{aligned}
p_{i} \geq & \left(\frac{f_{m}}{\mu_{B} K_{B}-\lambda_{B}}+\frac{\left(\left(K_{B}-1\right) \sqrt{\frac{\gamma_{C B R} \Omega_{i}}{\Omega_{\mathrm{D}}}}\right)}{\sqrt{2 \pi}\left(K_{B}-\frac{5}{4}\right)^{\frac{1}{2}}}\right) \\
& \times \pi(L-1) \sum_{n=0}^{L-2}\left(\begin{array}{c}
L-2 \\
n
\end{array}\right)(-1)^{n}(n+2)^{\frac{-3}{2}}
\end{aligned}
$$

In the interference system, if the normalized SNR is greater than or equal to the target SNR, the access probability of the best effort users is related with the number of channels as

$$
p_{i(B E)}=\operatorname{pr}\left(P_{i} \gamma_{i} \geq \gamma_{B E}\right)=\frac{1}{N_{t}-L}
$$


Substituting the above equation in Equation (43), the throughput of the best effort traffic is written as

$$
\begin{aligned}
T_{B E}= & \log _{2}\left[\frac { 1 } { p _ { i ( B E ) } } \left(\left(\left(\left[\frac{\alpha}{1+\alpha}\left(\frac{\left(1-\sigma_{E}^{2}\right)}{\delta}-1\right) *\left(\frac{1}{\sigma_{E}^{2}}\right)\right]\right)\right)\right.\right. \\
& \left.\left.+\sum_{l=L+1}^{N_{t}} \frac{1}{N_{r}-L}\right)\right]+\sum_{l=L+1}^{N_{t}}\left(\log _{2} e\right) \varphi\left(N_{r}-l+1\right)
\end{aligned}
$$

and we used the fact $E\left(\frac{1}{\gamma_{l}}\right)=\frac{1}{N_{r}-L}$ in the above equation. From the above equation, the throughput for best effort traffic is studied for various values of channel estimation error and the effect of interference on throughput from (46) is demonstrated in the results and discussion section.

\section{Results and Discussion}

The simulations are carried out for different MIMO systems. The numerical simulation consists of 1000 independent channel realizations of channel vectors and user distributions. Since in this proposed model, the transmitter is power constrained, the channel estimation error in this simulation is a decreasing function of signal to noise ratio [13] i.e. $\sigma_{E}^{2} \leq \sigma_{u}^{2}+\frac{\sigma_{n}^{2}}{\gamma_{B E}}$. From the initial population of $\mathrm{K}$ users, at any given time, a maximum of $K_{A}=8$ and $K_{B}=8$ users are considered. In performing simulation for interference scenarios, the simulation considered the worst case setting by considering the users are mutually interfering. The ratio of interference power to user fading power $\frac{\Omega_{i}}{\Omega_{\mathrm{D}}}$ averaged over the channel realizations is 0.2 and the transmission rate $c$ is set at minimum of $2 \mathrm{bits} / \mathrm{Hz}$. The performance of proposed power allocation is demonstrated for various number of receive and transmit antennas combinations $\left(N_{r} \times N_{t}\right)$. The important simulation parameters are shown in the Table 1.

In the Figure 2, the outage probability is compared for various values of total power. As the total power increases, we observe the following: (1) The outage probability decreases; (ii) The total available power can be allocated to the given number of channels and thus the users allocated to the channel will experience low outage; (iii) If $\alpha$ decreases, the total power allocated to the CBR users increases, and it results in lower outage probability there by providing higher QoS; (iv) If number of receiving antennas $N_{r}$ increases, the lower will be outage probability. These observations indicate that the proposed system reduces outage probability and also allocating the un-utilized 
Table 1 Simulation parameters

\begin{tabular}{lll}
\hline Variable & Parameter & Sim. Value \\
\hline$\gamma_{C B R}$ & CBR Target Signal to Noise Ratio & $6 \mathrm{~dB}$ \\
$\gamma_{B E}$ & BE Target Signal to Noise Ratio & $6 \mathrm{~dB}$ \\
$\alpha$ & Power Allocation Ratio & 0.1 and 0.9 \\
$\delta$ & SNR Loss Factor & $0.1 \mathrm{~dB}$ to $0.5 \mathrm{~dB}$ \\
$\sigma_{u}^{2}$ & Prediction Error & 0.03 \\
$\sigma_{n}^{2}$ & Measurement Error Caused by Noise & 0.05 and 0.1 \\
$L$ & Number of Channels & 1 to 8 \\
$P_{T o t a l}$ & Total Transmit Power & $1 \mathrm{~dB}$ to $20 \mathrm{~dB}$ \\
$T_{Q}$ & Maximum Queuing Delay & $10^{-3} \mathrm{Sec}$. \\
$f_{m}$ & Doppler Frequency & $40 \mathrm{~Hz}$ \\
$K$ & Total Number of Users & 20 \\
\hline
\end{tabular}

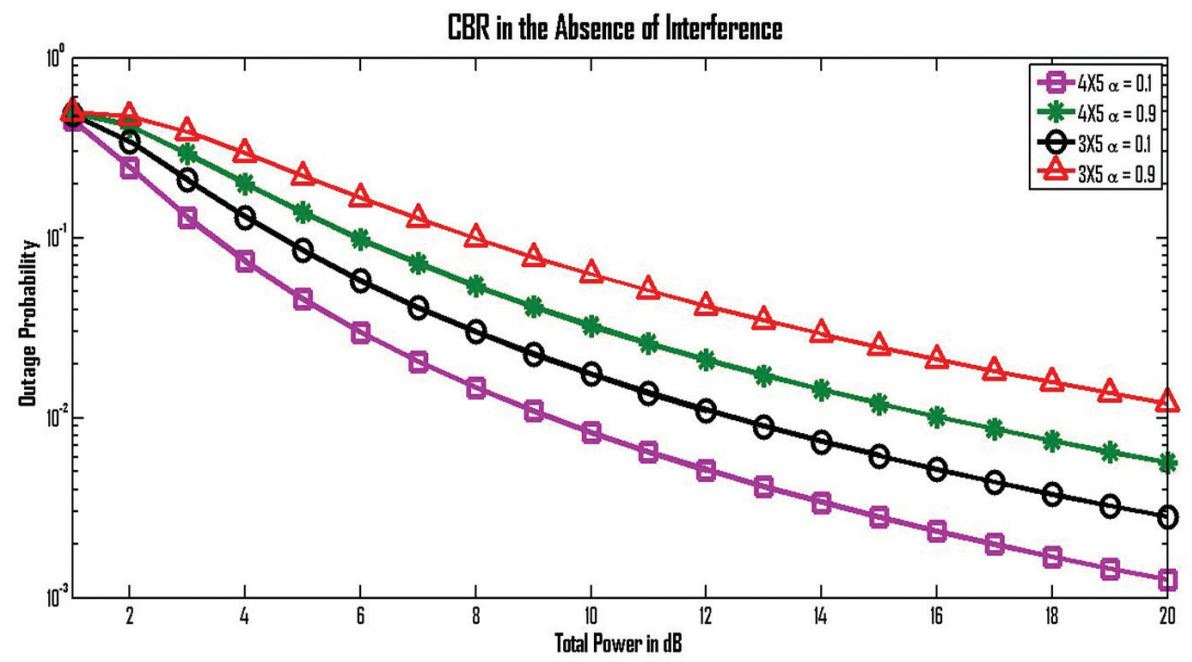

Figure 2 Outage probability against total power when $\mathrm{L}=2$.

power to the other traffic users for given total power budget. In the Figure 3, the outage probability is higher for given allocated total power because of strong interference. The outage probability in interference (Figure 3) is higher compared to without interference case (Figure 2) for the same system configuration i.e. one can make interesting observation by comparing Figure 2 and Figure 3, is that to serve the user with guaranteed QoS in interference, the total allocated power needs to be considerably increased compared to the 


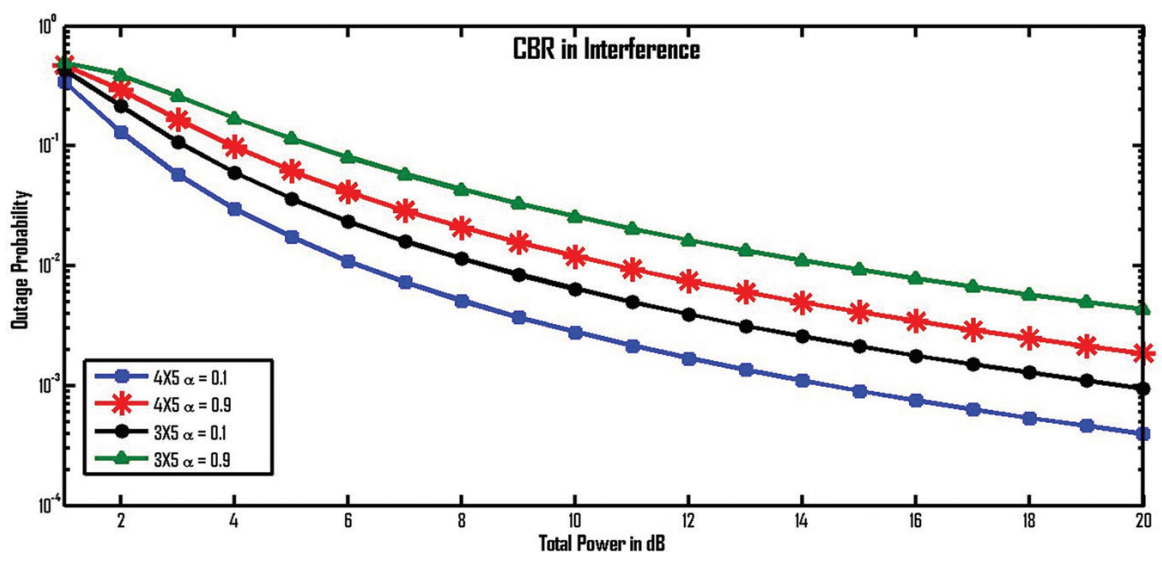

Figure 3 Outage probability against $P_{\text {Total }}$ in interference when $\mathrm{L}=2$.

without interference case. Generally it is required that the outage probability should be smaller than or equal to certain threshold value according to given QoS, any further decrease would not make significant impact.

To examine the behaviour of outage probability with channels, we evaluate the outage probability for $N_{r} \in\{3,4\}$ and $\alpha \in\{0.1,0.9\}$. The results are shown in Figure 4. The outage probability reduces as the number of channels increases. Moreover, we infer from the plot that if $N_{r}$ decreases and the value of $\alpha$ is maintained at the same value, the outage probability increases. This is because the allocated power can be used to increase the target SNR. The $3 \times 5$ system outage probability is higher compared to

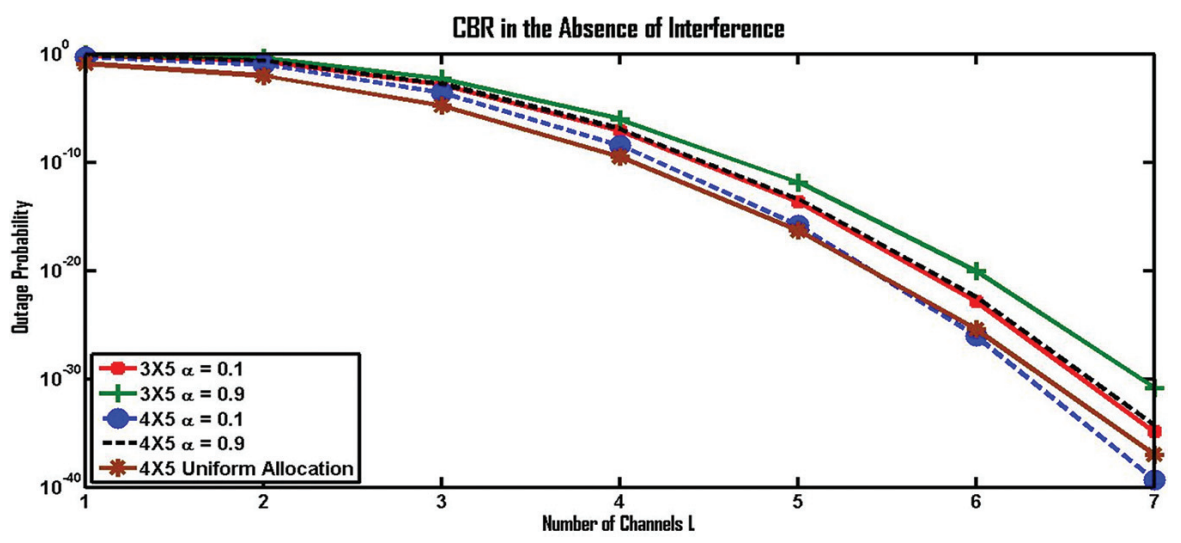

Figure 4 Outage probability against $L$ when $P_{\text {Total }}=20 \mathrm{~dB}$. 
the $4 \times 5$ system outage probability. Consequently, as L tends to increase, better outage probability of CBR is observed compared to the uniform power allocation.

The Figure 5 is the plot of outage probability with number of CBR users. Here if the number of user increases, the outage probability increases but at the same time, if the allocated power $P_{C B R}$ and $N_{r}$ increases, the outage probability decreases with number of users. It is interesting to notice that the outage probability is more a function of $\alpha$ than that of the number of receiving antennas. The Figure 6 demonstrates that (i) For higher $P_{\text {Total }}$ and $N_{r}$, the system produces higher throughput; (ii) If $\alpha$ is higher, the higher is the power allocated to the BE traffic and thus the throughput of the system will also increases.

Usually less power will be allocated to best-effort traffic and the impact of channel estimation is very important parameter to study the throughput in Best effort traffic. The throughput for best effort Traffic is analysed in Figure 7 for various values of variance obtained from the channel estimation error. It is clear from the Figure 7 that the throughput decreases as the predicted loss in SNR increases. It is inferred that if the value of $\sigma_{E}^{2}$ increases i.e. from $\sigma_{E}^{2}=$ 0.04 to $\sigma_{E}^{2}=0.05$, and simultaneously increasing the SNR loss factor, the proposed power allocation less likely to allocate more power to the best effort traffic users. Thus automatically, the throughput for the best effort traffic users decreases because required target SNR cannot be met in the presence of $\sigma_{E}^{2}$ and low $\alpha$. The decrease in throughput is clearly illustrated with SNR loss factor for different antenna combinations $\left(N_{r} \times N_{t}\right)$, power allocation ratio $\alpha$, and $\sigma_{E}^{2}$.

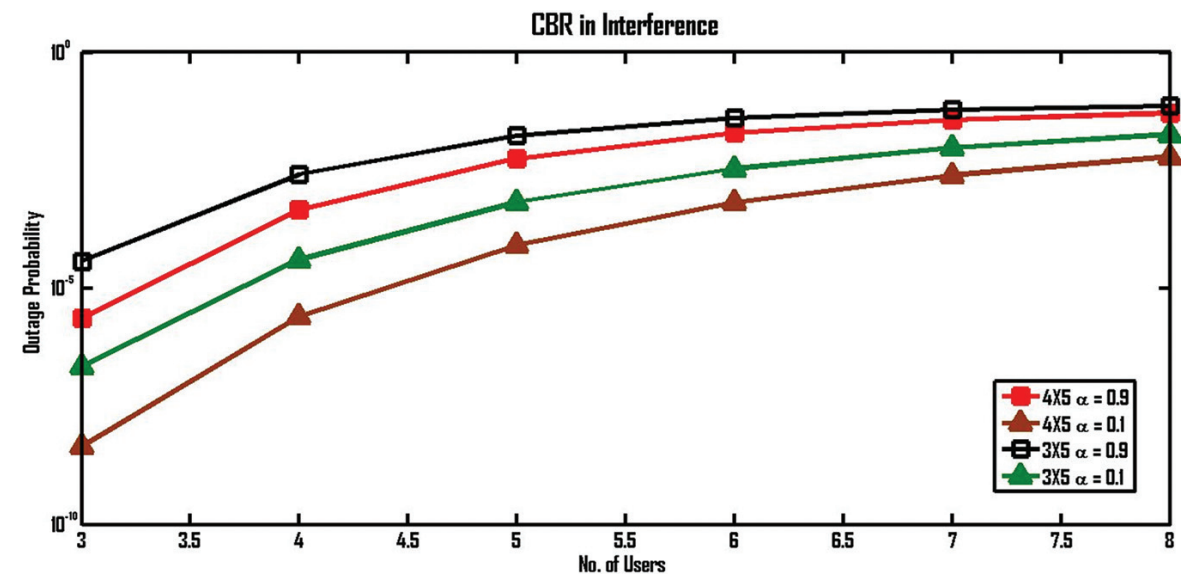

Figure 5 Outage probability versus number of CBR users $\mathrm{K}_{\mathrm{A}}$. 


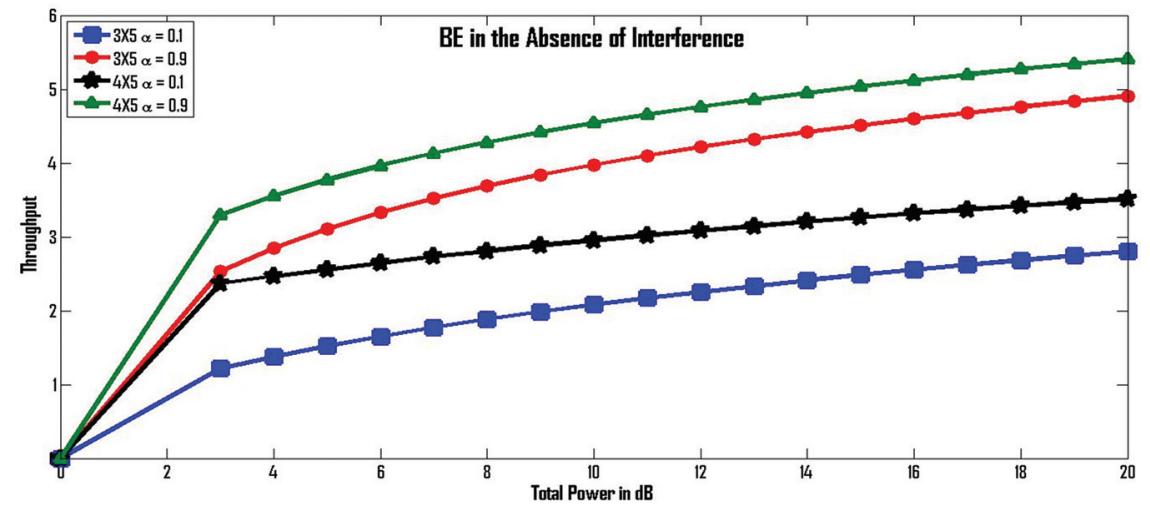

Figure 6 Throughput $(\mathrm{Bits} / \mathrm{sec} / \mathrm{Hz})$ vs. $P_{\text {Total }}$ when number $\mathrm{L}=2$.

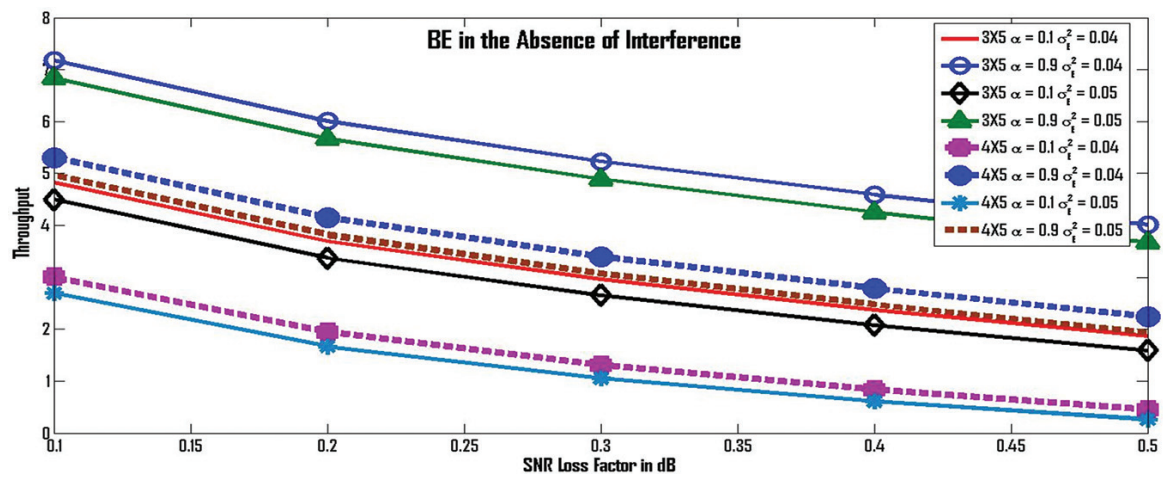

Figure 7 Throughput (Bits/sec/Hz) vs. SNR loss factor for different values of $\sigma_{E}^{2}$.

The Figure 8 demonstrates that the number of user increases, the throughput decreases and mainly the reduction in throughput is due the fact that the total power is fixed. This observation indicates that (i) Only less number of channels are available for the given total power to meet the growing number of users; (ii) As expected if $\sigma_{E}^{2}$ increases, the throughput decreases and it can easily be verified in the plot by comparing $\sigma_{E}^{2}=0.04$ and $\sigma_{E}^{2}=0.05$. Finally to investigate how the channel access is affected when the number of users increases, the probability of access is plotted with respect to threshold SNR in Figure 9. As the threshold SNR is increased, as expected the probability of access is decreasing. It is also clear from the graph (Figure 9) that the access probability is higher if more users trying to access the channel simultaneously. 


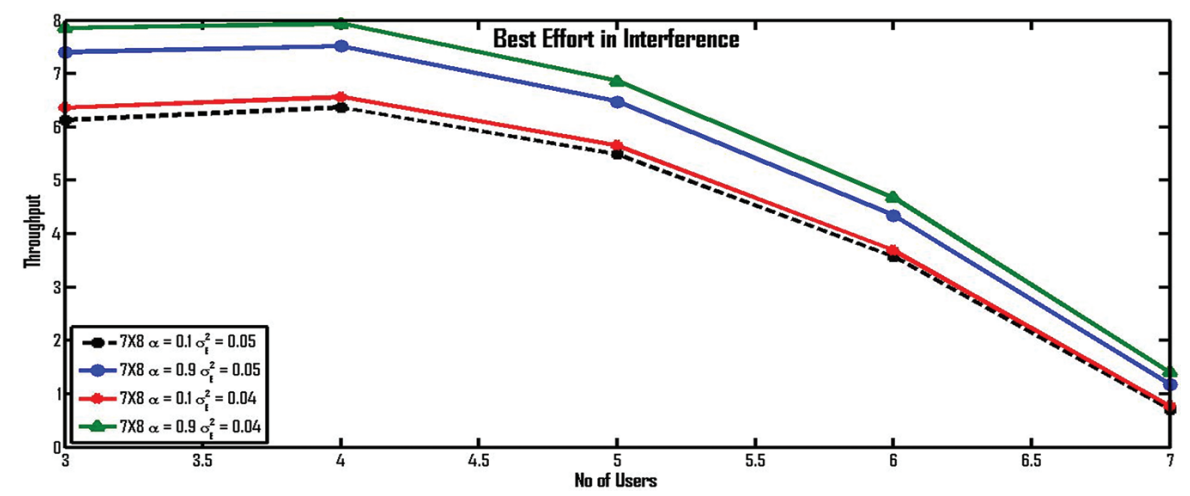

Figure 8 Throughput $(\mathrm{Bits} / \mathrm{sec} / \mathrm{Hz})$ vs. no. of users when $P_{\text {Total }}=20 \mathrm{~dB}$.

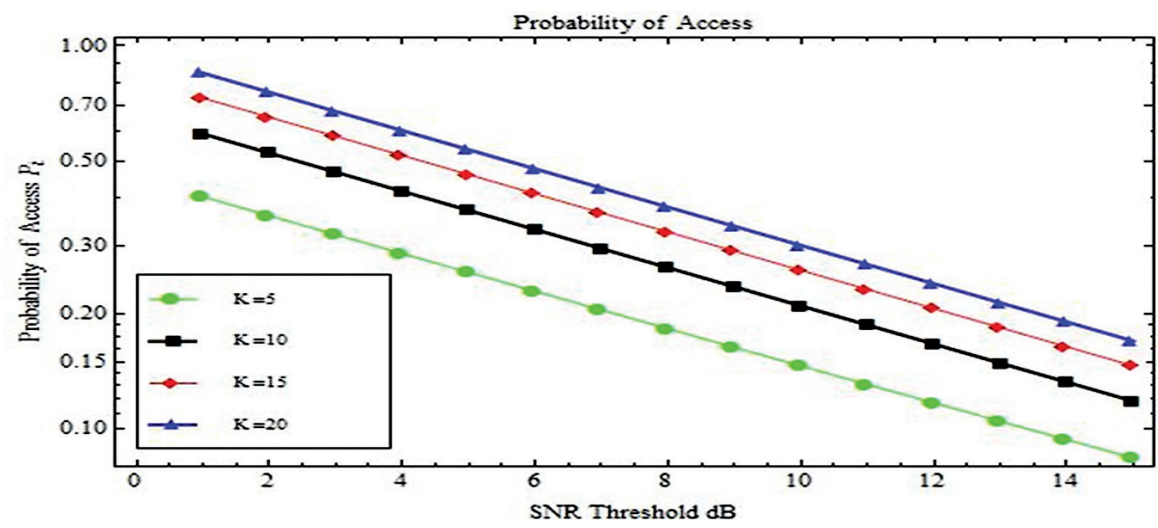

Figure 9 Probability of access to the SNR threshold for various $K$.

By evaluating the outage probability and throughput of service differentiated multiuser MIMO system, it is proved that our proposed method efficiently allocate power between $\mathrm{CBR}$ and $\mathrm{BE}$ users in order to guarantee the required QoS in interference as well as in without interference scenarios.

\section{Conclusions}

The multi-input multi-output system transmits traffics from different source nodes. Thus Power allocation is significant measure of quality and it is proved that it significantly affects the throughput and reduces the outage probability across sub channels of MIMO systems for QoS requirement. Closed form 
expressions are derived for optimal power utilization and to analyze the performance indices in both interference and non-interference regimes with probability access as one of the constraint.

The proposed Methodology can also be easily extended to heterogeneous MIMO networks and it will be interesting to study the power allocation by incorporating limited feedback delay.

\section{References}

[1] Goldsmith, A., Jafar, S. A., Jindal, N., and Vishwanath, S. (2003). Capacity limits of MIMO channels. IEEE J. Select. Areas Commun. 21, 684-702.

[2] Telatar, I. E. (1999). Capacity of multiple-antenna Gaussian channels. Eur. Trans. Telecomm. 10585-595.

[3] Biglieri, E., Caire, G., and Taricco, G. (2001). Limiting performance of block fading channels with multiple antennas. IEEE Trans. Inf. Theory 47, 1273-1289.

[4] Shiu, D., Foschini, G., Gans, M., and Kahn, J. (2000). Fading correlation and its effect on the capacity of multi-element antenna systems. IEEE Trans. Commun. 48, 502-513.

[5] Bolcskei, H., Nabar, R. U., Oyman, O., and Paulraj, A. J. (2006) Capacity scaling laws in MIMO relay networks. IEEE Trans. Wirel. Commun. 5, 1433-1444.

[6] Wang, N., and Blostein, S. D. (2007). Approximate minimum BER power allocation for MIMO spatial multiplexing systems. IEEE Trans. Commun. 55, 180-187.

[7] Choi, J. (2008). Power allocation for two different traffics in layered MIMO Systems. IEEE Trans. Commun. 7, 0, 3942-3950.

[8] Kim, I., Lee, K., and Chun, J. (2007). A MIMO antenna structure that combines transmit beamforming and spatial multiplexing. IEEE Trans. Wirel. Commun. 6, 775-779.

[9] Zheng, L., and Tse, D. N. C. (2013). Diversity and multiplexing: a fundamental trade-off in multiple-antenna channels. IEEE Trans. Inf. Theory 49, 1073- 1096.

[10] Li, L., Namuduri, K., and Fu, S. (2012). "Cooperative communication based on random beamforming strategy in wireless sensor networks," in Proceedings of Global Communications Conference, 4108-4113, California, USA. 
[11] Cheng, W., Zhang, X., and Zhang, H. (2014). Heterogonous statistical Qos provisioning for downlink transmissions over mobile cellular networks," in Proceedings of Global Communication Conference, 4622-4628, Austin, USA.

[12] Wang, P., Zhang, X., and Song, M. (2013). "Optimal stochastic subcarrier and power allocations for QoS-guaranteed services in OFDMA multicell cooperation networks," in Proceedings of IEEE International Conference on communications, 6449-6453, Budapest, Hungary.

[13] Yoo, T., and Goldsmith, A. (2006). Capacity and power allocation for fading MIMO channels with channel estimation error. IEEE Trans. Inf. Theory 52, 2203-2214.

[14] Yarg, L., and Alouini, M.-S. (2004). On the average outage rate and average outage duration of wireless communication systems with multiple cochannel interferers. IEEE Trans. Veh. Technol. 3, 1142-1153.

[15] KunjieXu, et al. (2014). Time varying performance analysis of multihop wireless networks with CBR Traffic. IEEE Trans. Veh. Technol. 63, 3397-3409.

[16] Goergen, N., Sabrina Lin, W., Liu, K. J. R., and Charles Clancy, T. (2011). Best effort cooperative relaying. IEEE Trans. wirel. commun. 10, 1833-1843.

[17] Xu, W., Tajer, A., Wang, X., and Alshomrani, S. (2014). Power allocation in MISO interference channels with stochastic CSIT. IEEE Trans. wirel. commun. 13, 1716-1727.

[18] Huang, K., and Lau, V. K. N. (2012). Stability and Delay of Zero-Forcing SDMA with Limited Feedback. IEEE Trans. Inf. Theory 58, 6499-6514.

[19] Yarg, L., and Alouini, M.-S. (2006). Performance analysis of multiuser selection diversity. IEEE Trans. Veh. Technol. 55, 1848-1861.

[20] Soulhli, O., and Ohtsuki, T. (2010). Joint Feedback and scheduling scheme for service-differentiated multuser MIMO Systems. IEEE Trans. Wirel. Commun. 9, 528-533.

[21] Femenias, G., Riera-Palou, F., and Thompson, J. S. (2016). "Robust scheduling and resource allocation in the downlink of spatially correlated MIMO-OFDMA wireless systems with imperfect CSIT. IEEE Trans. Veh. Technol. 65, 614-629.

[22] J. Choi, Kim, Y.-H., Lee, J.-H., Bahk, S., and Kim, S.-C. (2012). User throughput estimation for the PF scheduling algorithm under MIMO channel environments. IEEE Wirel. Commun., Lett. 1, 528-531.

[23] Tse, D., and Viswanath, P. (2005) Fundamentals of Wireless Communication. Cambridge: University Press. 
[24] Chih-Liang chen, Wayne E. Stark, and Sau-Gee Chen, S.-G. (2011). Energy Efficiency Tradeoff in MIMO Multi-Hop Wireless Networks. IEEE J. Select. Areas Commun. 29, 1537-1546.

[25] Hwang, D.-K., and Jaehoon Whang, R. (2009). Efficient Detection Ordering Scheme for MIMO Transmission Using Power Control. IEEE Sig. Process. Lett. 16, 715-718.

\section{Biographies}

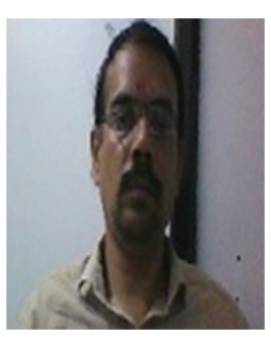

S. Balaji received Master of Technology from National Institute of Technology, Tiruchirapalli, India in 2002. Currently he is working as Assistant Professor (Selection Grade) in the School of Electrical Engineering (SELECT), VIT University, India. He has published more than 15 research papers in the area of wireless communication in International and National Level Journals and Conferences. His areas of interest include MIMO, OFDM, and Wireless Communication with emphasis on Signal Processing.

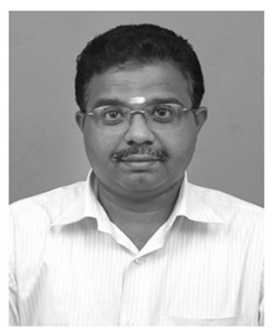

L. Nithyanandan received Bachelor of Engineering from University of Madras in 1992, Master of Technology in 1999 and Ph.D. degree in 2006 from Pondicherry University. He is working as Professor of Electronics and Communication Engineering, Pondicherry Engineering College, Puducherry, 
India. He is a gold medalist in PG and has been awarded with chief minister medal for his outstanding performance in PG. He has more than 50 publications in National/International conferences and Journals. His areas of interest include Sensor Networks, Telemedicine, Spread Spectrum Techniques and Wireless Communication.

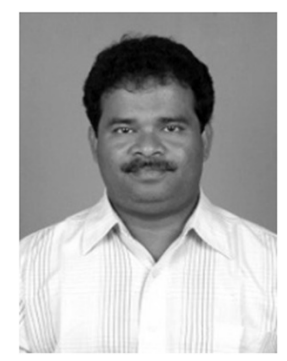

P. S. Mallick, (M'03, SM'10) received M.S degree from the University of Chittagong, Bangladesh and Ph.D from Jadavpur University, India. He worked 4.5 years in a Sweden based electronics industry named IAAB Electronics as a Technical Head. He has 10 years of Teaching experience where he led various research teams and developed "Online Lab in Microelectronics", "Monte Carlo Simulator," and "Electric Fencer". His current area of research interest includes Optoelectronic materials and Devices, Nanoscale CMOS and Nanoelectronics. He has published more than 50 research papers in different Journals and Conferences of International repute and authored a book on Matlab and Simulink. At Present Dr. P. S. Mallick is working for School of Electrical Engineering, VIT University, Tamil Nadu, India, as a Senior Professor. He has received the prestigious Jawaharlal Nehru Scholarship in 1998 for his doctoral research work. He is one of the enlisted technical innovators of India in 2007. 